\title{
Targeted Raman Imaging of Cells by Graphene Oxide-Based Hybrids
}

Zhenyu Zhang, Meng Wang, Dongliang Gao, Da Luo, Qinghai Liu, Juan Yang, and Yan $\mathrm{Li}^{*}$

Beijing National Laboratory for Molecular Science, Key Laboratory for the Physics and Chemistry of Nanodevices, State Key Laboratory of Rare Earth Materials Chemistry and Applications, College of Chemistry and Molecular Engineering, Peking University, Beijing 100871, China

E-mail: yanli@pku.edu.cn 

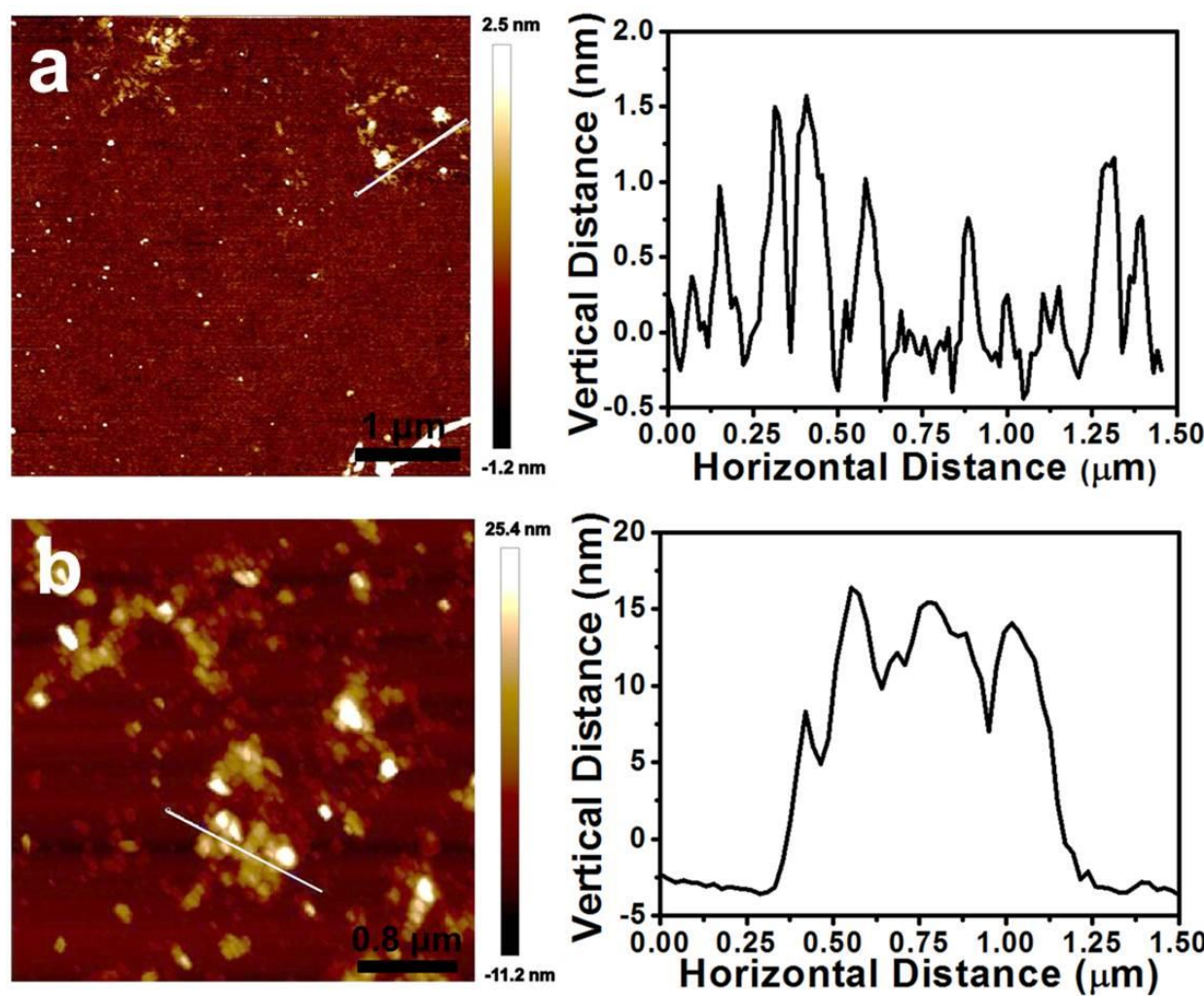

Figure S1. (a) Left: AFM image of GO/Pt; Right: height distribution of the white line in the left image. (b) Left: AFM image of GO/AuNP; Right: height distribution of the white line in the left image.
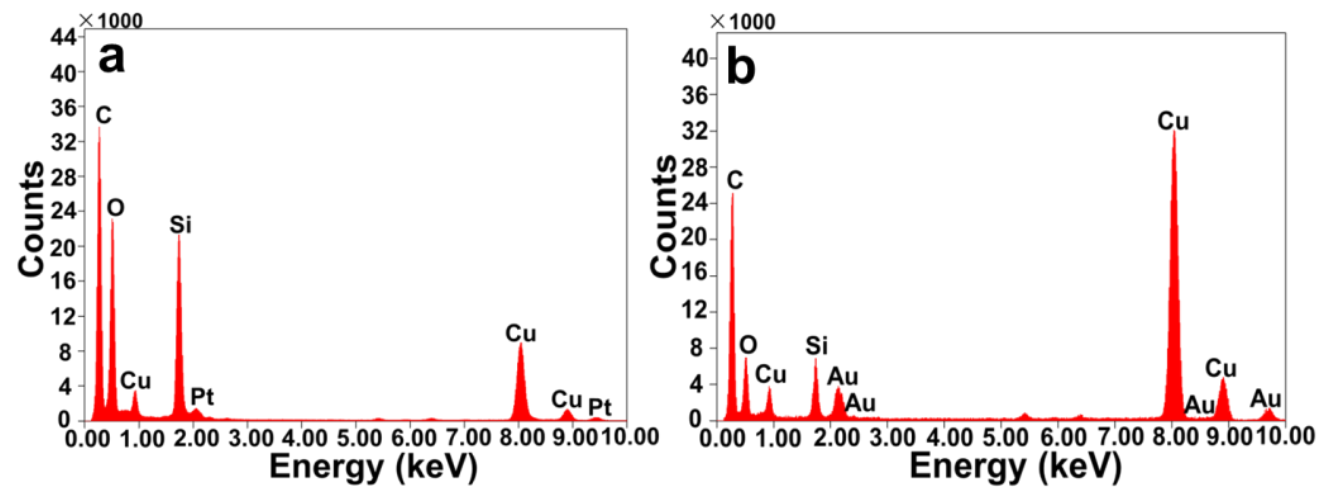

Figure S2. EDAX spectra of GO/Pt (a) and GO/AuNP (b) hybrids. 


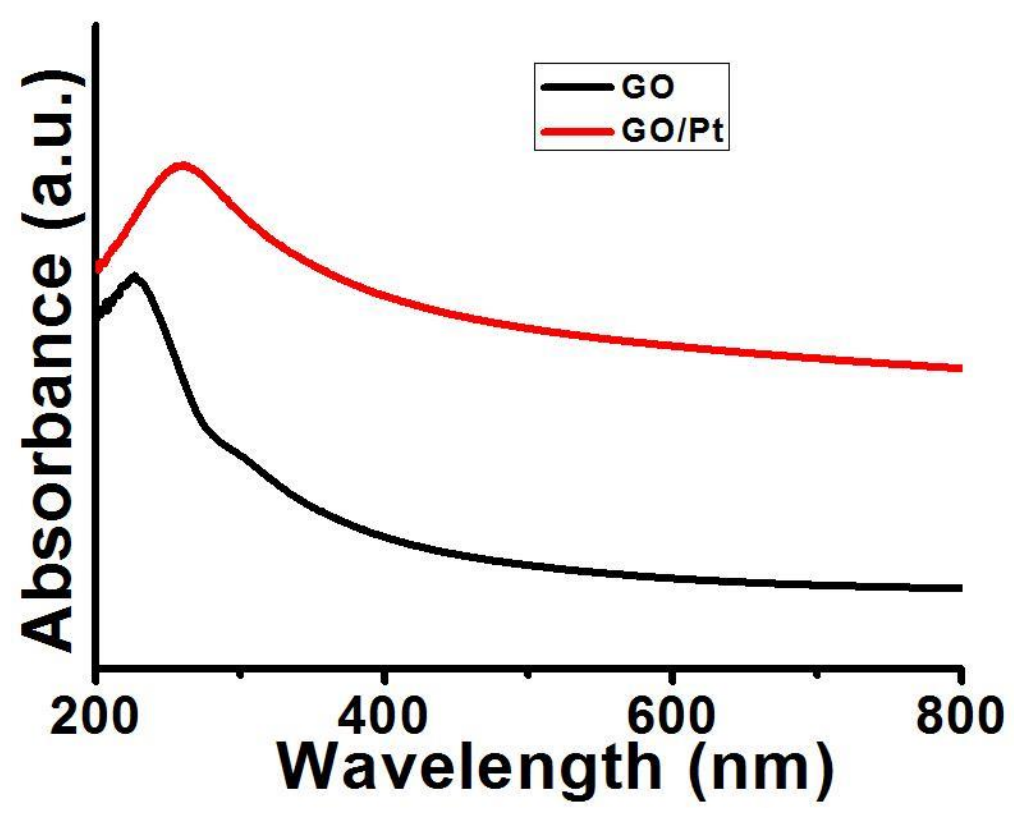

Figure S3. UV-Vis absorption spectra of GO and GO/Pt.

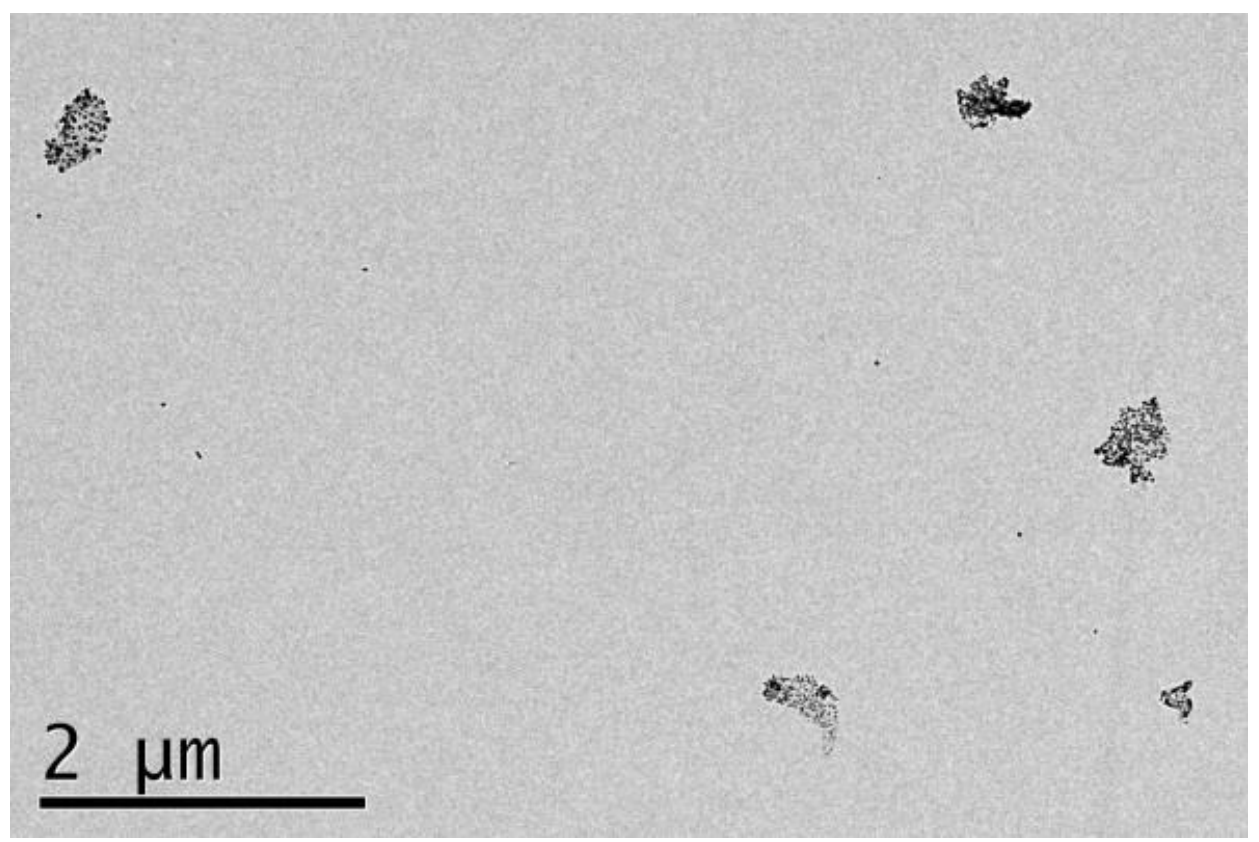

Figure S4. TEM image of GO/AuNP hybrids. 


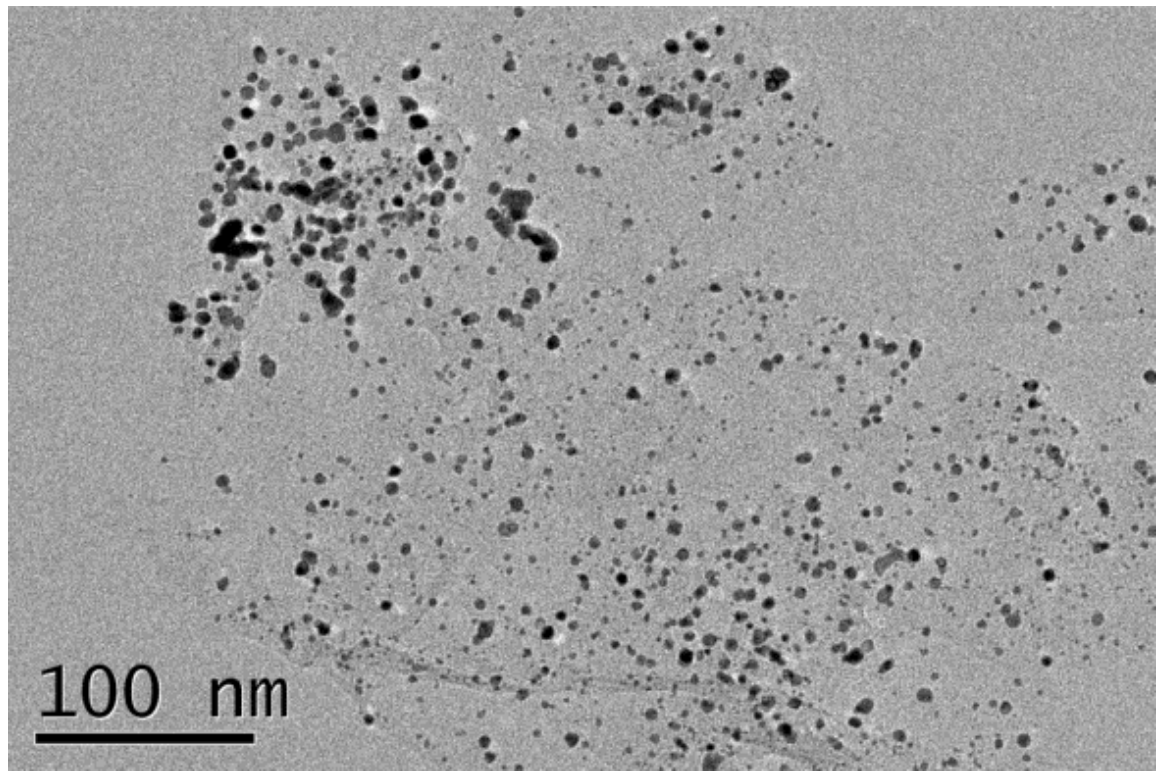

Figure S5. TEM image of GO/AuNP hybrids prepared using less $\mathrm{HAuCl}_{4}$.
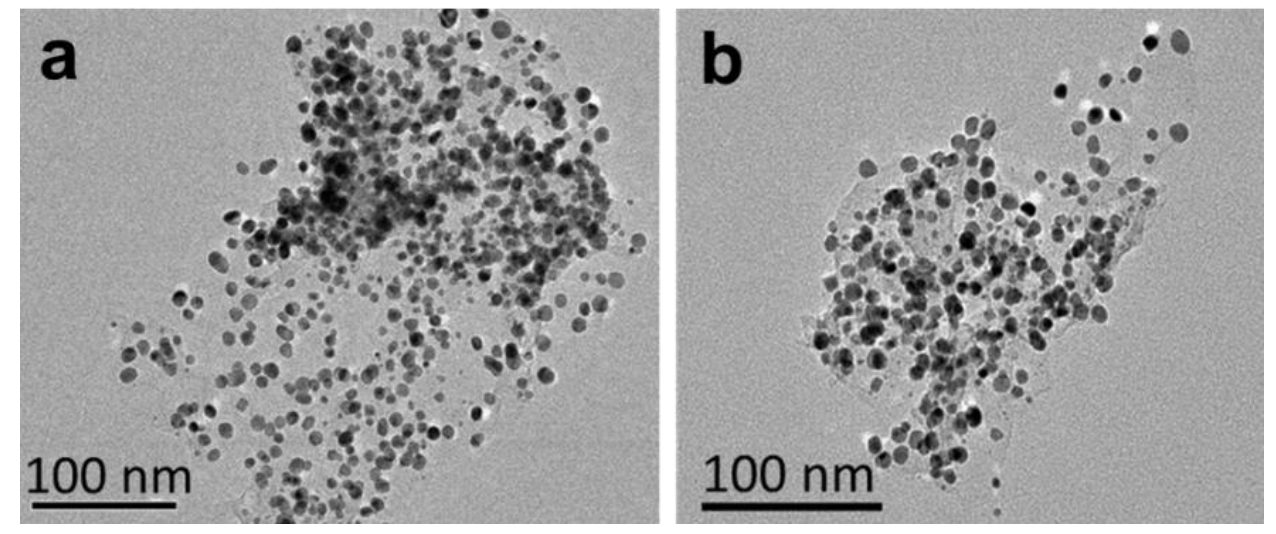

Figure S6. TEM images of GO/AuNP/TGA (a) and GO/AuNP/FA (b) hybrids. 


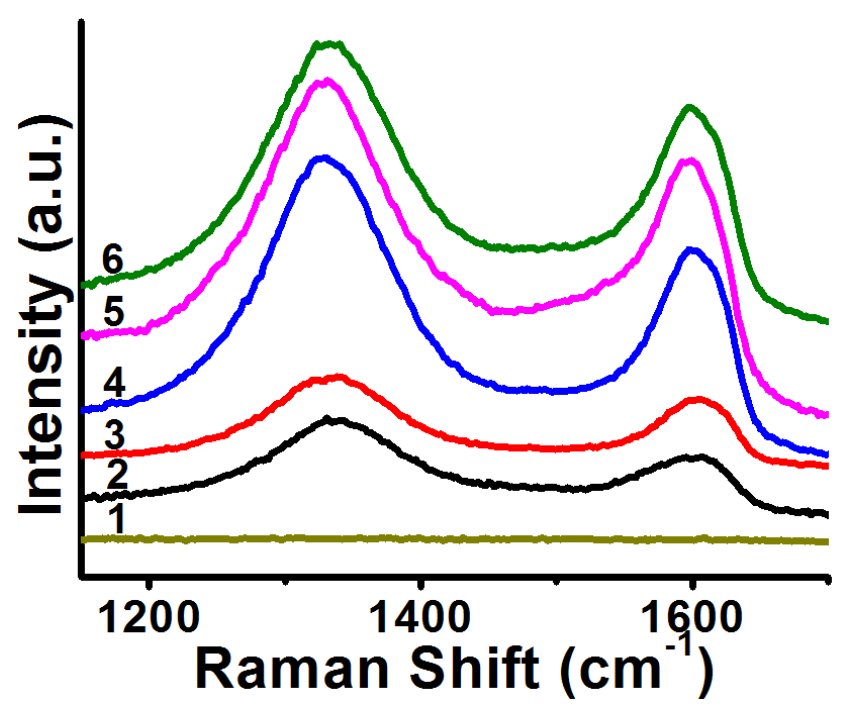

Figure S7. Raman spectra of FA (1), GO (2), GO/Pt (3), GO/AuNP (4), GO/AuNP/TGA (5), and GO/AuNP/FA (6) hybrids.

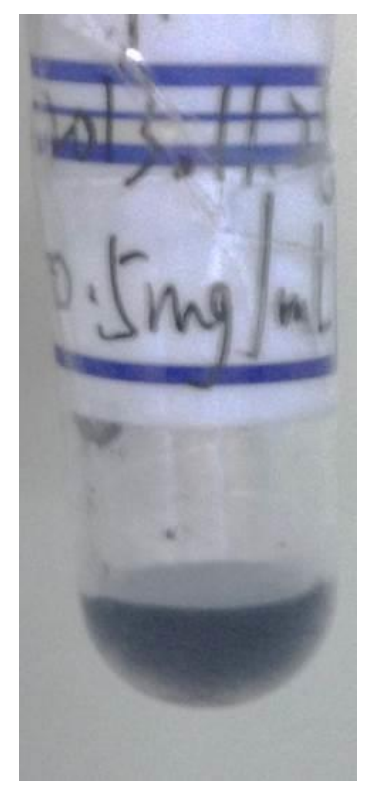

Figure S8. The dispersion stability of GO/AuNP/FA hybrids prepared in 2013. 


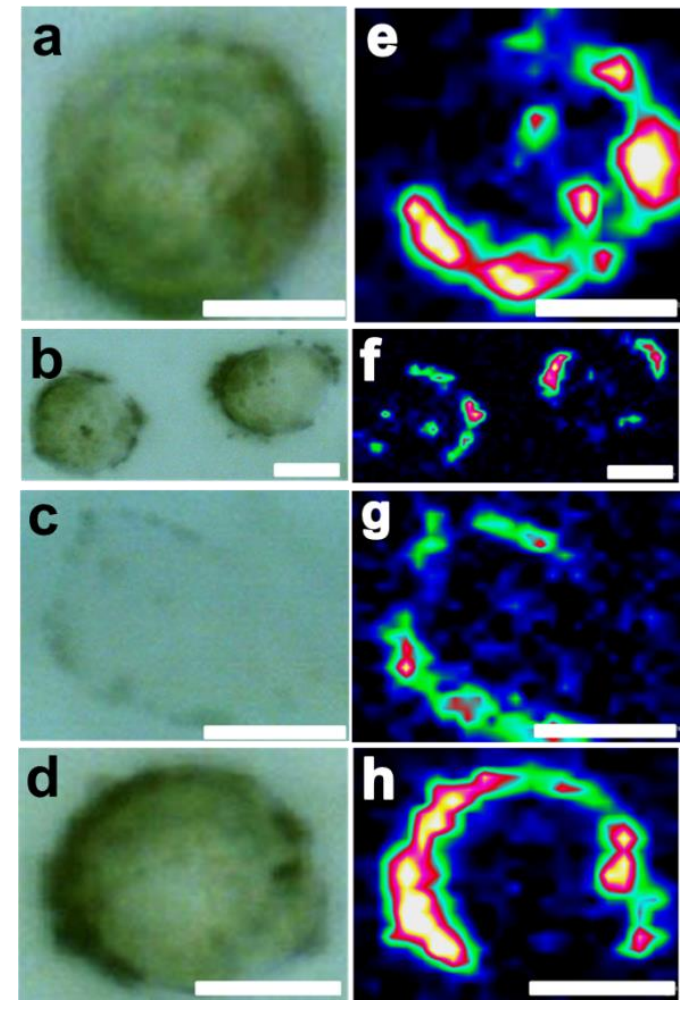

Figure S9. Optical (a-d) and Raman (e-h) images of cells. Different batches of experiments carried out by $\mathrm{FR}^{+} \mathrm{HeLa}$ cells incubated with GO/AuNP/FA hybrids diluted using FA RPMI 1640 media. Scale bar is $10 \mu \mathrm{m}$. 